\title{
ON AN INFINITE SYSTEM OF COMPATIBILITY CONDITIONS ALONG A SHOCK RAY
}

By RENUKA RAVINDRAN and PHOOLAN PRASAD

(Department of Mathematics, Indian Institute of Science, Bangalore 560012, India)

[Received 4 February 1991. Revise 20 February 1992]

\section{SUMMAR Y}

To study the position and strength of a shock discontinuity as it propagates into a medium at rest, an infinite system of compatibility conditions can be derived. Each of these involves derivatives of a single flow variable and is in the form of a transport equation along shock rays. For two-dimensional shock propagation, the first two compatibility conditions are derived in detail.

\section{Introduction}

THE idea of a shock discontinuity and the jump relations across it was first introduced by Stokes (1) as early as 1848 and the Rankine-Hugoniot jump relations across a shock have been known for about 120 years (Rankine (2), Hugoniot (3)). However, the possibility of deriving an infinite system of compatibility conditions on a shock manifold in space-time was proposed only recently by Grinfel'd (4) and Maslov (5). These conditions involve relations between a single variable (say, the density) and its derivatives behind the shock and are in the form of transport equations along shock rays. A clear definition of shock rays was given by Prasad (6). Roy and Ravindran (7) proved a general uniqueness theorem confirming that the definition of shock rays is mathematically consistent.

Grinfel'd derived the compatibility conditions for nonlinear elasticity and Maslov those for the gas-dynamic equations. Maslov actually developed a general theory for a system of first-order conservation laws using the theory of distributions and derived the conditions in a very elegant way from the results of a uniqueness lemma. However, since he was interested in establishing certain results for a weak shock, he considered the motion of an isentropic gas and thus ignored the energy equation. The first compatibility condition for the full set of conservation laws of gas dynamics was derived by Srinivasan and Prasad (8) using Maslov's lemma.

A careful study of the proof of Maslov's uniqueness lemma shows that it can be derived only under extra assumptions (see (A.2) and (A.4) in the Appendix). In general these assumptions are not true. These difficulties in the proof of the lemma do not influence the first compatibility condition derived by Srinivasan and Prasad (8), but the second and higher compatibility conditions need to be corrected. Elucidation of the mistake in Maslov's lemma and derivation of the 
correct compatibility conditions have now become important for the following reasons.

(i) By truncating the infinite system of compatibility conditions for a model conservation law, we have recently developed a new theory of shock dynamics $(9,10)$, whose derivation is mathematically convincing (Prasad (11)) and which gives extremely accurate results. This theory gives not only the shock strength and shock position but also a few spatial derivatives behind the shock so that we can approximately contruct the unknown solution at any time by using a finite Taylor series. There is considerable saving of computer time in this procedure compared to the usual finite-difference method.

(ii) Derivation of the compatibility conditions is extremely complex and not only requires very careful long mathematical calculations but also utmost care in the change of the order of certain differential operators since they do not commute. It is necessary to derive as many compatibility conditions as possible in a very compact form for ready reference and use. The new theory of shock dynamics with two and three compatibility conditions derived along the shock path for a plane shock has been used by Lazarev, Prasad and Singh (12) to obtain the solution of the one-dimensional acceleration-piston problem with non-zero initial piston speed. The solution of this problem, when the piston speed is not small (leading to a strong shock), is almost impossible by any method other than a purely numerical method. We derive the compatibility conditions in the rest of the paper, and we have commented on the proof of Maslov's lemma in Appendix A.

\section{The first compatibility condition}

We consider the propagation of a shock front in a polytropic gas with $\gamma$ as the constant ratio of the specific heats. For simplicity, we assume that the motion is two-dimensional, that there exists only one smooth shock manifold $\Omega$ in space-time $\left(\mathbb{R}^{3}\right)$ and that the fluid velocity $q=(u, v)$, pressure $p$ and density $\rho$ are $C^{\infty}\left(\mathbf{R}^{3}\right)$ functions except for a discontinuity of the first kind on $\Omega$. We further assume that the shock front propagates into a gas in a uniform state and at rest ahead of the shock, that is, $\mathbf{q}_{a}=(0,0), p_{a}=p_{0}$ and $\rho_{a}=\rho_{0}$, where $p_{0}$ and $\rho_{0}$ are constants. The state behind the shock manifold $\Omega$ is denoted by $q_{b}=\left(u_{b}, v_{b}\right), p_{b}$ and $\rho_{b}$. These functions, defined only in the domain behind the shock, are then extended as $C^{\infty}$ functions on the whole of $\mathbb{R}^{3}$. The extended functions $q_{b}, p_{b}$ and $\rho_{b}$ are non-unique in the domain ahead of the shock.

Let $s(x, y, t)=0$ denote the equation of the shock surface $\Omega$ in space-time. For a shock in an ideal gas with constant specific heats, it has been shown (Prasad (6), Roy and Ravindran (7)) that the function $s$ can be obtained by solving any one of a number of shock-manifold partial differential equations (SME), one of which is

$$
s_{1}+C\left(s_{x}^{2}+s_{y}^{2}\right)^{\frac{1}{4}}=0
$$


with

$$
C^{2}=\frac{\rho_{b}}{\rho_{0}} \frac{p_{b}-p_{0}}{\rho_{b}-\rho_{0}}
$$

where $p_{b}$ and $\rho_{b}$ are in $C^{\infty}\left(\mathbb{R}^{3}\right)$.

We define a function

$$
\mu=\frac{\rho_{b}-\rho_{0}}{\rho_{0}} \in C^{\infty}\left(\mathbf{R}^{3}\right)
$$

whose value on $\Omega$ represents the shock strength. A shock front denoted by $\Omega_{\text {, }}$ at any time $t$ is a curve in the $(x, y)$-plane and is given by $s(x, y, t)=0$ in which $t$ appears as a parameter. The Rankine-Hugoniot conditions give the following relations on $\Omega$ :

$$
\begin{gathered}
\left.C\right|_{\Omega}=a_{0}\left(\frac{2(1+\mu)}{2-(\gamma-1) \mu}\right)^{\ddagger}, \\
\left.p_{b}\right|_{\Omega}=\left.p_{0} \frac{2+(\gamma+1) \mu}{2-(\gamma-1) \mu}\right|_{\Omega}
\end{gathered}
$$

and

$$
\left.\left(u_{b}, v_{b}\right)\right|_{\Omega}=\left.\frac{\mu C}{1+\mu}\left(N_{1}, N_{2}\right)\right|_{\Omega}
$$

where $a_{0}^{2}=\gamma p_{0} / \rho_{0}$ and $\left(N_{1}, N_{2}\right)=(\cos \Theta, \sin \Theta)$ is the unit normal to the shock front $\Omega_{t}$. The relations (2.4) to (2.6) are not valid in $\mathbb{R}^{3}$ but only on $\Omega$. The SME is valid in $\mathbb{R}^{3}$ and hence it is possible to define $\Theta$ in $\mathbb{R}^{3}$ with the help of the relation

$$
\left(N_{1}, N_{2}\right)=\frac{\left(s_{x}, s_{y}\right)}{\left(s_{x}^{2}+s_{y}^{2}\right)^{\frac{1}{t}}}
$$

The derivative

$$
\frac{d}{d t}=\frac{\partial}{\partial t}+N_{1} C \frac{\partial}{\partial x}+N_{2} C \frac{\partial}{\partial y}
$$

represents the time rate of change as we move along a characteristic curve of the equation (2.1). When $C$ is evaluated on $\Omega$, then this is the time rate of change along a shock ray and is an interior derivative in $\Omega$. We denote the normal and the tangential derivatives for the shock front by $\partial / \partial N, \partial / \partial T$, that is,

$$
\frac{\partial}{\partial N}=N_{1} \frac{\partial}{\partial x}+N_{2} \frac{\partial}{\partial y}, \quad \frac{\partial}{\partial T}=N_{2} \frac{\partial}{\partial x}-N_{1} \frac{\partial}{\partial y} .
$$

We also denote the normal and tangential components of the fluid velocity by 
$A$ and $B$ respectively; then

and

$$
\left.A\right|_{\mathbf{\Omega}}=\left.\frac{\mu C}{1+\mu}\right|_{\Omega}
$$

$$
\left.B\right|_{\mathbf{\Omega}}=0 .
$$

We write the equations of conservation of mass, momentum and energy behind the shock as follows (after dropping the subscript $b$ ):

$\frac{d \rho}{d t}+(A-C) \frac{\partial \rho}{\partial N}+\rho\left(\frac{\partial A}{\partial N}-A \frac{\partial \Theta}{\partial T}\right)+\left[B \frac{\partial \rho}{\partial T}+\rho\left(\frac{\partial B}{\partial T}+B \frac{\partial \Theta}{\partial N}\right)\right]=0$

$\frac{d A}{d t}+(A-C) \frac{\partial A}{\partial N}+\frac{1}{\rho} \frac{\partial p}{\partial N}+B\left[\frac{d \Theta}{d t}+(A-C) \frac{\partial \Theta}{\partial N}+\frac{\partial A}{\partial T}+B \frac{\partial \Theta}{\partial T}\right]=0$,

$$
\begin{aligned}
& \frac{d \Theta}{d t}+(A-C) \frac{\partial \Theta}{\partial N}-\left(\frac{A-C}{A}\right) \frac{\partial B}{\partial N}-\frac{1}{\rho A} \frac{\partial p}{\partial T} \\
&-\frac{1}{A}\left[\frac{d B}{d t}+B \frac{\partial B}{\partial T}-A B \frac{\partial \Theta}{\partial T}\right]=0, \\
& \frac{d p}{d t}+(A-C) \frac{\partial p}{\partial N}-\frac{\gamma p}{\rho}\left(\frac{d \rho}{d t}+(A-C) \frac{\partial \rho}{\partial N}\right)+B\left[\frac{\partial p}{\partial T}-\frac{\gamma p}{\rho} \frac{\partial \rho}{\partial T}\right]=0 .
\end{aligned}
$$

In (2.16) $C, p, A, \rho$ on $\Omega$ are known functions of $\mu$ (see (2.3), (2.6)) and the derivative $d / d t$ is an interior derivative in $\Omega$, so that (2.3) to (2.6) can be used to determine $d p / d t$ and $d A / d t$ in terms of $\mu$ and $d \mu / d t$ :

$$
\begin{gathered}
\left.\frac{d p}{d t}\right|_{\mathbf{\Omega}}=\left.p_{0} \frac{4 \gamma}{\{2-(\gamma-1) \mu\}^{2}} \frac{d \mu}{d t}\right|_{\mathbf{\Omega}}, \\
\left.\frac{d A}{d t}\right|_{\mathbf{\Omega}}=\left.C \frac{4+3 \mu-\gamma \mu}{2(1+\mu)^{2}(2-(\gamma-1) \mu)} \frac{d \mu}{d t}\right|_{\Omega},
\end{gathered}
$$

where we have used the fact that $N_{1}^{2}+N_{2}^{2}=1$. This leads to the first 
compatibility condition on $\Omega$ along the shock rays:

$$
\frac{1}{C} \frac{d \mu}{d t}=\mu \frac{Q}{S}\left[2 \frac{\partial \Theta}{\partial T}-\frac{(\gamma+1)}{1+\mu} \frac{\partial \mu}{\partial N}\right] \text { on } \Omega,
$$

where

$$
Q=2-\mu(\gamma-1), \quad S=8+5 \mu-3 \mu \gamma+\mu^{2}\left(\gamma^{2}-1\right) .
$$

The characteristic equations for the shock-manifold partial differential equation (2.1) (see Prasad (6)) give the shock-ray equations

$$
\frac{d x}{d t}=N_{1} C, \quad \frac{d y}{d t}=N_{2} C
$$

and

$$
\frac{d \Theta}{d t}=\frac{\partial C}{\partial T}
$$

On $\Omega$, equation (2.22) reduces to

$$
\frac{1}{C} \frac{d \Theta}{d t}=\frac{\gamma+1}{2 Q(1+\mu)} \frac{\partial \mu}{\partial T}
$$

Equations (2.21) (restricted to $\Omega$ ), (2.23) and (2.19) form a coupled system of four equations for the position $(x, y)$ of the shock, the inclination $\Theta$ of the normal to the shock and the shock strength $\mu$. Since $\partial / \partial T$ is an interior derivative on $\Omega, \partial \mu / \partial T$ and $\partial \Theta / \partial T$ are known on $\Omega$ if $\mu$ and $\Theta$ are known on $\Omega$. The system, however, is not closed due to the presence of the normal derivative $\partial \mu / \partial N$ in (2.19). The presence of such a normal derivative rendering the system incomplete is typical of the compatibility conditions on $\Omega$ (see Ravindran and Prasad (10)).

\section{The second compatibility condition}

To obtain the second compatibility condition, each of the equations of mass, momentum and energy is differentiated with respect to $\partial / \partial N$. The terms are then rearranged to give the following three equations on $\Omega$ (corresponding to equations (2.12), (2.13), (2.15) in the first compatibility condition):

$$
\begin{gathered}
\frac{d}{d t}\left(\frac{\partial \rho}{\partial N}\right)+(A-C) \frac{\partial^{2} \rho}{\partial N^{2}}+\rho \frac{\partial^{2} A}{\partial N^{2}}+\rho \frac{\partial}{\partial T}\left(\frac{\partial B}{\partial N}\right)-\rho A \frac{\partial}{\partial T}\left(\frac{\partial \Theta}{\partial N}\right) \\
+\left(2 \frac{\partial A}{\partial N}-A \frac{\partial \Theta}{\partial T}\right) \frac{\partial \rho}{\partial N}+\left(\frac{d \Theta}{d t}-C \frac{\partial \Theta}{\partial N}+\frac{\partial B}{\partial N}\right) \frac{\partial \rho}{\partial T} \\
+2 \rho \frac{\partial B}{\partial N} \frac{\partial \Theta}{\partial N}-\rho A\left(\frac{\partial \Theta}{\partial N}\right)^{2}-\rho A\left(\frac{\partial \Theta}{\partial T}\right)^{2}-\rho \frac{\partial A}{\partial N} \frac{\partial \Theta}{\partial T}=0 \text { on } \Omega,
\end{gathered}
$$




$$
\begin{aligned}
& \frac{d}{d t}\left(\frac{\partial A}{\partial N}\right)+(A-C) \frac{\partial^{2} A}{\partial N^{2}}+\frac{1}{\rho} \frac{\partial^{2} p}{\partial N^{2}}+\left(\frac{d \Theta}{d t}-C \frac{\partial \Theta}{\partial N}+\frac{\partial B}{\partial N}\right) \frac{\partial A}{\partial T} \\
& +\left(\frac{\partial A}{\partial N}\right)^{2}+\frac{\partial B}{\partial N}\left(\frac{d \Theta}{d t}+(A-C) \frac{\partial \Theta}{\partial N}\right)+\frac{1}{\rho} \frac{\partial \rho}{\partial N}\left(\frac{d A}{d t}+(A-C) \frac{\partial A}{\partial N}\right)=0 \text { on } \Omega
\end{aligned}
$$

and

$$
\begin{array}{r}
\frac{d}{d t}\left(\frac{\partial p}{\partial N}\right)-\frac{\gamma p}{\rho} \frac{d}{d t}\left(\frac{\partial \rho}{\partial N}\right)+(A-C)\left(\frac{\partial^{2} p}{\partial N^{2}}-\frac{\gamma p \partial^{2} \rho}{\rho \partial N^{2}}\right)+\left(\frac{d \Theta}{d t}-C \frac{\partial \Theta}{\partial N}\right) \\
\times\left(\frac{\partial p}{\partial T}-\frac{\gamma p}{\rho} \frac{\partial \rho}{\partial T}\right)+\frac{\partial A}{\partial N}\left(\frac{\partial p}{\partial N}-\frac{\gamma p}{\rho} \frac{\partial \rho}{\partial N}\right)+\frac{\partial B}{\partial N}\left(\frac{\partial p}{\partial T}-\frac{\gamma p}{\rho} \frac{\partial \rho}{\partial T}\right) \\
-\gamma\left[\frac{1}{\rho} \frac{\partial p}{\partial N}-\frac{p}{\rho^{2}} \frac{\partial \rho}{\partial N}\right]\left[\frac{d \rho}{d t}+(A-C) \frac{\partial \rho}{\partial N}\right]=0 \text { on } \Omega .
\end{array}
$$

The fourth equation, corresponding to.(2.14) in the first compatibility condition, is of interest only for evaluating the third compatibility condition. The expressions for $\partial A / \partial N, \partial B / \partial N$ and $\partial p / \partial N$ on $\Omega$ can be obtained in terms of $\mu$, $\partial \mu / \partial T, \partial \mu / \partial N, \partial \Theta / \partial T, \partial \Theta / \partial N$ from (2.13) to (2.15) and (2.19) and (2.23). These expressions are then substituted for all interior derivatives in the equations (3.1) to (3.3). The terms $\partial^{2} A / \partial N^{2}$ and $\partial^{2} p / \partial N^{2}$ are eliminated from the three equations (3.1) to (3.3), to give the following equation:

$$
\begin{aligned}
\frac{1}{C} \frac{d}{d t}\left(\frac{\partial \mu}{\partial N}\right)=-\frac{1}{\zeta_{1}} & {\left[\frac{\mu(1+\gamma)}{2(1+\mu)^{3}} \frac{\partial^{2} \mu}{\partial N^{2}}+\zeta_{2}\left(\frac{\partial \mu}{\partial N}\right)^{2}+\zeta_{3} \frac{\partial \mu}{\partial N} \frac{\partial \Theta}{\partial T}\right.} \\
& \left.+\zeta_{4}\left(\frac{\partial \Theta}{\partial T}\right)^{2}+\zeta_{5} \frac{\partial^{2} \mu}{\partial T^{2}}+\zeta_{6}\left(\frac{\partial \mu}{\partial T}\right)^{2}+\zeta_{7} \frac{\partial \mu}{\partial T} \frac{\partial \Theta}{\partial N}\right],
\end{aligned}
$$

where the coefficients $\zeta_{1}, \zeta_{2}, \ldots, \zeta_{7}$ are known functions of $\gamma$ and $\mu$. These coefficients are expressed in terms of $\delta_{1}, \delta_{2}, \ldots, \delta_{6}$ and $\beta_{1}, \beta_{2}, \ldots, \beta_{6}$, which appear in the equations

$$
\begin{gathered}
\frac{1}{C} \frac{d}{d t}\left(\frac{\partial p}{\partial N}\right)=\rho_{0} C\left[\beta_{1} \frac{d}{d t}\left(\frac{\partial \mu}{\partial N}\right)+C \beta_{2}\left(\frac{\partial \mu}{\partial N}\right)^{2}+C \beta_{3} \frac{\partial \mu}{\partial N} \frac{\partial \Theta}{\partial T}+C \beta_{4}\left(\frac{\partial \Theta}{\partial T}\right)^{2}\right. \\
\left.+C \beta_{5} \frac{\partial^{2} \mu}{\partial T^{2}}+C \beta_{6}\left(\frac{\partial \mu}{\partial T}\right)^{2}\right] \text { on } \Omega
\end{gathered}
$$

and

$$
\begin{aligned}
\frac{1}{C} \frac{d}{d t}\left(\frac{\partial A}{\partial N}\right)= & \delta_{1} \frac{d}{d t}\left(\frac{\partial \mu}{\partial N}\right)+C \delta_{2}\left(\frac{\partial \mu}{\partial N}\right)^{2}+C \delta_{3} \frac{\partial \mu}{\partial N} \frac{\partial \Theta}{\partial T}+C \delta_{4}\left(\frac{\partial \Theta}{\partial T}\right)^{2} \\
& +C \delta_{5} \frac{\partial^{2} \mu}{\partial T^{2}}+C \delta_{6}\left(\frac{\partial \mu}{\partial T}\right)^{2} \text { on } \Omega
\end{aligned}
$$


The expressions for all these coefficients are given in Appendix B.

We derive a quantity $\mu_{i}(i=1,2,3, \ldots)$ by

$$
\mu_{i}=\left\{\sum_{r+s=i}{ }^{i} C_{r} N_{1}^{r} N_{2}^{s} \frac{\partial^{i} \mu}{\partial x^{\prime} \partial y^{y}}\right\} .
$$

Obviously $\mu_{1}=\partial \mu / \partial N$ and it is easy to verify that

$$
\frac{\partial^{2} \mu}{\partial N^{2}}+\frac{\partial \mu}{\partial T} \frac{\partial \Theta}{\partial N}=\mu_{2}
$$

The second compatibility condition (3.4) finally becomes

$$
\begin{aligned}
\frac{1}{C} \frac{d \mu_{1}}{d t}=-\frac{1}{\zeta_{1}} & {\left[\frac{\mu(1+\gamma)}{2(1+\mu)^{3}} \mu_{2}+\zeta_{2} \mu_{1}^{2}+\zeta_{3} \mu_{1} \frac{\partial \Theta}{\partial T}\right.} \\
& \left.+\zeta_{4}\left(\frac{\partial \Theta}{\partial T}\right)^{2}+\zeta_{5} \frac{\partial^{2} \mu}{\partial T^{2}}+\zeta_{6}\left(\frac{\partial \mu}{\partial T}\right)^{2}\right] \text { on } \Omega .
\end{aligned}
$$

The first compatibility condition (2.11) was originally incomplete due to the appearance of $\Theta$ and $\partial \mu / \partial N=\mu_{1}$ on the right-hand side. The shock-ray equations partially removed the incompleteness by providing the equation (2.23) for $\Theta$. The search for an equation for $\mu_{1}$ led to the second compatibility condition (3.8) which is also incomplete due to the presence of $\mu_{2}$. The search for an equation for $\mu_{2}$ leads to the third compatibility condition containing $\mu_{3}$ (Grinfel'd (4)) and the process could continue, leading to an infinite system of compatibility conditions.

Application of the above two compatibility conditions to the new theory of shock dynamics (Prasad and Ravindran (9)) will be presented in subsequent publications.

\section{REFERENCES}

1. G. G. STOKEs, Phil. Mag. 33 (1848) 349-356.

2. W. J. M. RANKINE, Trans. R. Soc. 160 (1870) 277-288.

3. H. HUGONIOT, J. Ecole polytechnique 58 (1889) 1-125.

4. M. A. GrinfeL'D, J. appl. Math. Mech. 42 (1978) 958-977.

5. V. P. MASLOV, J. Sov. Math. 13 (1978): English tr. (1980) 119-163.

6. P. Prasad, Acta Mech. 45 (1982) 163-176.

7. R. RoY and R. Ravindran, ibid. 73 (1988) 239-244.

8. R. SRinivasan and P. Prasad, Proc. Ind. Acad. Sci. (Math. Sci.) 94 (1985) 27-42.

9. P. Prasad and R. Ravindran, Appl. Math. Letters 3 (1990) 107-109.

10. R. Ravindran and P. Prasad, ibid. 3 (1990) 77-81.

11. P. Prasad, Proc. Ind. Acad. Sci. (Math. Sci.) 100 (1990) 87-92.

12. M. P. Lazarev, P. Prasad and S. Singh, One-dimensional accelerating piston problem with nonzero piston speed (under preparation). 


\section{APPENDIX A}

Comments on the proof of Maslov's uniqueness lemma (5)

A statement of the lemma and an elucidation of the exact points where the proof of the lemma does not hold would take considerable space. Therefore, we shall mention the reasons briefly. Let $\Omega: s\left(x_{1}, x_{2}, x_{3}, t\right)=0$ be the shock manifold in space-time and $\Omega_{1}$ be its section by a $t$-constant plane. Let $g^{k}$ (after dropping the subscript from $g_{j}^{k}$ of Maslov) be a function in $C^{\infty}\left(\mathbf{R}^{4}\right)$. With $g^{k}$ we associate the normal derivatives $\left.g_{i}^{k}\right|_{\Omega}$ on the surface $\Omega$ by

$$
\left.\left.g_{i}^{k}(\mathbf{x}, t)\right|_{\Omega} \equiv\langle\nabla s, \nabla\rangle g^{k}\right|_{\Omega}=\left.\left\{\left(s_{x_{1}} \frac{\partial}{\partial x_{1}}+s_{x_{2}} \frac{\partial}{\partial x_{2}}+s_{x_{3}} \frac{\partial}{\partial x_{s}}\right)^{t} g^{k}\right\}\right|_{\Omega} .
$$

We extend any function $\left.g_{i}^{k}(x, t)\right|_{\Omega}$ defined on $\Omega$ in a smooth manner on the whole of $\mathbb{P}^{4}$ so that the extended function $g_{i}^{k}(x, t)$ is constant along the trajectories of the field $\langle\nabla s, \nabla\rangle$, that is, $\langle\nabla s, \nabla\rangle g_{i}^{k}=0, i=0,1,2, \ldots$ Note that $\left.g^{k}(\mathbf{x}, t)\right|_{\Omega}=\left.g_{0}^{k}(x, t)\right|_{\Omega}$ but $g^{k}(\mathbf{x}, t) \neq g_{0}^{k}(\mathbf{x}, t)$ in $\mathbb{R}^{4}$. In the proof of the lemma, the function $g$ is expanded in a neighbourhood of the surface of discontinuity $\Omega$ by a Taylor series with remainder as

$$
g^{k}(\mathbf{x}, t)=\sum_{i=0}^{n+1} g_{i}^{k}(\mathbf{x}, t) \frac{s^{i}(\mathbf{x}, t)}{i !}+R_{n+2}^{k} \frac{s^{n+2}}{(n+2) !}, \quad \mathbf{x}=\left(x_{1}, x_{2}, x_{3}\right) .
$$

The expansion (A.1) is valid only if the function $s$ can be so chosen that

$$
|\nabla s|=1 \text { in the neighbourhood of } \Omega \text { in } \mathbb{R}^{4} \text {. }
$$

This is, in general, not possible since $s$ will also have to satisfy the shock-manifold partial differential equation.

The final step in the proof of the lemma is to use the operator $\langle\nabla s, \nabla\rangle^{n}(n=1,2, \ldots)$ on a function $h$ which is of the form

$$
h=\sum_{k=1}^{m}\left\langle e^{k}, \nabla_{t \mathbf{x}}\right\rangle g^{k}(\mathbf{x}, t)
$$

where $e^{k}$ is a fixed four-dimensional vector and

with

$$
\nabla_{t x}=\left(\frac{\partial}{\partial t}, \nabla\right)
$$

$$
\nabla=\left(\frac{\partial}{\partial x_{1}}, \frac{\partial}{\partial x_{2}}, \frac{\partial}{\partial x_{3}}\right)
$$

If the function $h$ vanishes identically, it follows that $\left.\langle\nabla s, \nabla\rangle^{\star} h\right|_{\Omega}=0$ from which Maslov deduces the result of his lemma, namely that $\left.h_{n}\right|_{\Omega}=0$. While doing so he uses the incorrect identity

$$
\langle\nabla s, \nabla\rangle^{n}\left\langle e^{k}, \nabla_{t x}\right\rangle g_{i}^{k}(\mathbf{x}, t)=\left\langle e^{k}, \nabla_{t x}\right\rangle\langle\nabla s, \nabla\rangle^{n} g_{l}^{k}(\mathbf{x}, t), \quad i=1,2, \ldots, n+1 .
$$

This does not hold as we can see for the particular case when $e^{k}=(1,0,0,0)$ :

$$
\begin{aligned}
\langle\nabla s, \nabla\rangle^{*}\left\langle e^{k}, \nabla_{i \mathbf{x}}\right\rangle g_{i}^{k}(\mathbf{x}, t) & =\langle\nabla s, \nabla\rangle^{*} \frac{\partial g_{i}^{k}}{\partial t} \\
& =\langle\nabla s, \nabla\rangle^{*-1}\left[\frac{\partial}{\partial t}\left\{\langle\nabla s, \nabla\rangle g_{i}^{k}\right\}-\left\langle\nabla s_{t}, \nabla\right\rangle g_{i}^{k}\right] .
\end{aligned}
$$


Since $\nabla s_{t}$ and $\nabla s$ need not be parallel, the final term on the right-hand side need not vanish, that is, interchange of the order of operators $\langle\nabla s, \nabla\rangle^{n}$ and $\left\langle e^{k}, \nabla_{i x}\right\rangle$ leads to further terms on the right-hand side of (A.4).

\section{APPENDIX B}

Expressions for the coefficients in equations (3.4) to (3.6)

$$
\begin{aligned}
R= & 4+3 \mu-\mu \gamma+\mu^{2}\left(\gamma^{2}-1\right), \\
T= & 4+6 \mu-2 \mu \gamma+3 \mu^{2}\left(\gamma^{2}-1\right), \\
U= & 5-3 \gamma+2 \mu\left(\gamma^{2}-1\right), \\
\beta_{1}= & \frac{1}{2(1+\mu)^{2}}\left[2+\mu(\gamma+1)-\frac{\mu^{3}(\gamma+1)\left(\gamma^{2}-1\right)}{S}\right], \\
\beta_{2}= & \frac{\mu(\gamma+1)}{(1+\mu)^{2}} \frac{Q}{S}\left\{\beta_{1}\left(2-\frac{\gamma+1}{Q}\right)+\frac{\gamma+1}{2(1+\mu) S}\left(3 \mu^{2}\left(\gamma^{2}-1\right)-S-\frac{\mu^{3}\left(\gamma^{2}-1\right) U}{S}\right)\right\}, \\
\beta_{3}= & \frac{\mu Q}{S(1+\mu)}\left\{\beta_{1}\left(-4+2 \frac{\gamma+1}{Q}\right)-\frac{\gamma+1}{(1+\mu) S}\left(6 \mu^{2}\left(\gamma^{2}-1\right)-S-2 \mu^{3} \frac{\left(\gamma^{2}-1\right) U}{S}\right.\right. \\
& \left.\left.+\frac{\left(\gamma^{2}-1\right)(\gamma-1) \mu^{3}}{Q}\right)\right\}, \\
\beta_{4}= & \frac{\mu^{3}\left(\gamma^{2}-1\right)}{(1+\mu) S^{2}}\left\{S+6 Q+2 \mu(\gamma-1)-\frac{2 \mu U Q}{S}\right\}, \\
\beta_{5}= & \frac{\mu^{3}\left(\gamma^{2}-1\right)(\gamma+1)}{2(1+\mu)^{2} Q S}, \\
\beta_{6}= & -\frac{\mu^{3}\left(\gamma^{2}-1\right)(\gamma+1)\{5-3 \gamma-4 \mu(\gamma-1)\}}{4(1+\mu)^{3} Q^{2} S},
\end{aligned}
$$

and

$$
\begin{aligned}
\delta_{1}= & \frac{1}{2(1+\mu)^{2}}\left\{2+\mu(\gamma+1)-\frac{\mu(\gamma+1) R}{S}\right\}, \\
\delta_{2}= & \frac{\mu(\gamma+1) Q}{(1+\mu)^{2} S}\left\{\delta_{1}\left(2-\frac{\gamma+1}{2 Q}\right)+\frac{\gamma+1}{2(1+\mu) S}\left(T-S-\frac{\mu R U}{S}\right)\right\}, \\
\delta_{3}= & \frac{\mu Q}{(1+\mu) S}\left\{\frac{\gamma+1}{\mu+1}-4 \delta_{1}+\frac{\gamma+1}{2 Q}\left(2 \delta_{1}-\frac{\gamma+1}{(\mu+1)^{2}} \frac{\mu R}{S}\right)+\frac{\mu(\gamma+1) R}{(1+\mu)^{2} S}\right. \\
& \left.-2 \frac{(\gamma+1)}{(1+\mu) S}\left(T-\frac{\mu R U}{S}\right)\right\}, \\
\delta_{4}= & \frac{\mu}{(1+\mu) S}\left\{R+\frac{2 Q}{S}\left(T-\frac{\mu R U}{S}\right)+\frac{2 \mu Q R}{(1+\mu) S}\left(-1+\frac{\gamma+1}{2 Q}\right)\right\},
\end{aligned}
$$




$$
\begin{aligned}
& \delta_{5}=\frac{(1+\gamma) \mu R}{2(1+\mu)^{2} Q S} \\
& \delta_{6}=-\frac{(\gamma+1)\{5-3 \gamma-4 \mu(\gamma-1)\} \mu R}{4(1+\mu)^{3} Q^{2} S}
\end{aligned}
$$

and

$$
\begin{aligned}
\zeta_{1}= & \delta_{1}+\beta_{1}-\frac{\mu(1+\gamma)}{2(1+\mu)^{2}}, \\
\zeta_{2}= & \delta_{2}+\beta_{2}+\frac{(\gamma+1)}{4(1+\mu)^{4}}\{(2+\mu)(2+\mu(1+\gamma)) \\
& +\frac{\mu}{S}[8(1+\mu)(\gamma-1)-2(4+3 \mu-\mu \gamma)(4+\mu+\mu \gamma) \\
& \left.-\mu^{2}\left(\gamma^{2}-1\right)(6+4 \gamma+3 \mu(\gamma+1))\right]+\frac{\mu^{2}(\gamma+1)}{S^{2}} \\
& \left.\times\left[R\left(R+\mu^{2}\left(\gamma^{2}-1\right)\right)-2 \gamma \mu^{2}\left(\gamma^{2}-1\right) Q\right]\right\} \\
\zeta_{3}= & \delta_{3}+\beta_{3}+\frac{1}{2(1+\mu)^{3}}\left\{-(2+\mu(3+\gamma))+\frac{\mu}{S}[(4+3 \mu-\mu \gamma)(9+\gamma+2 \mu+2 \mu \gamma)\right. \\
& \left.-8(1+\mu)(\gamma-1)+\mu^{2}\left(\gamma^{2}-1\right)(7+5 \gamma+3 \mu+3 \mu \gamma)\right] \\
& \left.-\frac{2 \mu^{2}(\gamma+1)}{S^{2}}\left[R\left(R+\mu^{2}\left(\gamma^{2}-1\right)\right)-2 \gamma \mu^{2}\left(\gamma^{2}-1\right) Q\right]\right\}, \\
\zeta_{4}= & \delta_{4}+\beta_{4}-\frac{1}{(1+\mu)^{2}}\left\{\mu+\frac{\mu R}{S}-\frac{\mu^{2}}{S^{2}}\left[\left(R+\mu^{2}\left(\gamma^{2}-1\right)\right) R-2 \gamma \mu^{2}\left(\gamma^{2}-1\right) Q\right]\right\}, \\
\zeta_{9}= & \delta_{9}+\beta_{9}+\frac{4-\mu(1+\gamma)}{2 Q(1+\mu)^{2}}, \\
\zeta_{6}= & \delta_{6}+\beta_{6}+\frac{(\gamma+1)}{4(1+\mu)^{3} Q^{2}}\left\{20+\mu(3-5 \gamma)+\mu^{2}\left(\gamma^{2}+4 \gamma-9\right)-\mu^{3}\left(\gamma^{2}-1\right)\right\} . \\
&
\end{aligned}
$$

